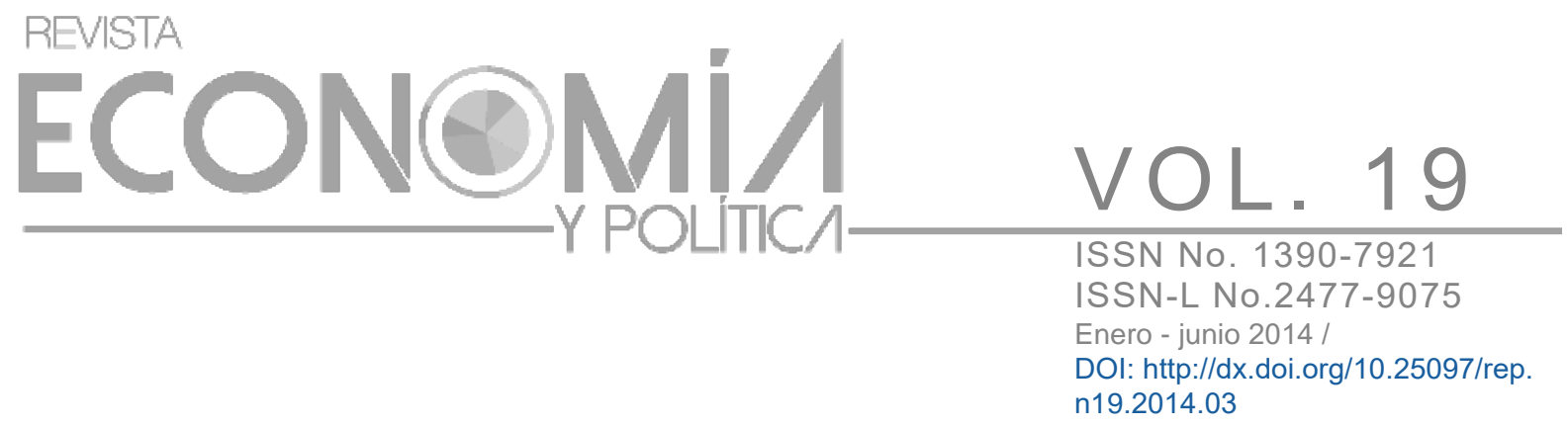

\title{
UN MODELO TGARCH CON UNA DISTRIBUCIÓN T DE STUDENT ASIMÉTRICA Y LAS HIPOTESIS DE RACIONALIDAD DE LOS INVERSIONISTAS BURSÁTILES EN LATINOAMÉRICA
}

\section{A TGARCH MODEL WITH AN ASYMMETRIC STUDENT'S T DISTRIBUTION AND THE RATIONALITY HYPOTHESES OF STOCK INVESTORS IN LATIN AMERICA}

\author{
Arturo Lorenzo Valdés** \\ Universidad de las Américas, Puebla. \\ arturo.lorenzo@udlap.mx
}

\author{
Antonio Ruiz Porras*** \\ Universidad de Guadalajara, CUCEA \\ antoniop@cucea.udg.mx
}

\section{Resumen}

Proponemos un modelo ARCH de tipo TGARCH con una distribución t de Student asimétrica. El mismo se construye usando la metodología de Fernández y Steel (1998) y el modelo TGARCH tradicional desarrollado por Zakoian (1994). El modelo se usa para describir series de rendimientos bursátiles y para evaluar la validez de las hipótesis de racionalidad en Latinoamérica. Los resultados sugieren que: 1) Las series de rendimientos analizadas pueden describirse adecuadamente con el modelo propuesto; 2) la hipótesis de racionalidad de Samuelson es consistente con la evidencia de los mercados de Argentina, Brasil, Chile, Colombia y México; 3) la hipótesis tradicional de racionalidad es consistente con la evidencia de Perú; y 4) las volatilidades estimadas mediante el modelo propuesto son mayores que las estimadas mediante el modelo TGARCH tradicional durante el periodo 2008-2009.

Palabras clave: Distribución de Densidad, $\mathrm{t}$ de Student Asimétrica, TGARCH, Rendimientos Bursátiles, Latinoamérica

\begin{abstract}
We propose an ARCH model of the TGARCH type with an asymmetric Student's t distribution. It is built using the methodology of Fernandez and Steel (1998) and the traditional TGARCH model developed by Zakoian (1994). The model is used to describe series of stock market returns and to assess the validity of the rationality hypotheses in Latin America. The results suggest that: 1) The series can be described adequately with the proposed model; (2) the Samuelson's rationality hypothesis is consistent with the evidence of the markets of Argentina, Brazil, Chile, Colombia and Mexico; 3) the traditional rationality hypothesis is consistent with the evidence of Peru; and (4) the volatility estimated with the proposed model are higher than those estimated with the traditional TGARCH model over the period 2008-2009.
\end{abstract}

Keywords: Density Distribution, Asymmetric t-Student, TGARCH, Stock Market Returns, Latin America

JEL: C22, G10, F30

\footnotetext{
** Email: arturo.lorenzo@udlap.mx Departamento de Finanzas y Contaduría, Universidad de las Américas Puebla. Sta. Catarina Mártir, 72820, Cholula, Puebla, México.

*** Email: antoniop@cucea.udg.mx Departamento de Métodos Cuantitativos. Universidad de Guadalajara, CUCEA. Periferico Norte 799, Núcleo Universitario Los Belenes, 45100, Zapopan, Jalisco, México.
} 


\section{UN MODELO TGARCH CON UNA DISTRIBUCIÓN T DE STUDENT \\ ASIMÉTRICA Y LAS HIPOTESIS DE RACIONALIDAD DE LOS \\ INVERSIONISTAS BURSÁTILES EN LATINOAMÉRICA}

\section{Introducción}

En la literatura econométrica muchos esfuerzos han sido desarrollados para estudiar las dinámicas de las series de rendimientos bursátiles. Estos esfuerzos se justifican porque las series suelen tener características muy difíciles de modelar. Las series suelen tener comportamientos muy volátiles, curtosis excesivas, clusters de volatilidad, volatilidades no constantes y distribuciones no normales. En añadidura a las anteriores, en las series bursátiles suele ocurrir que la volatilidad presente depende de "shocks informacionales". Particularmente, se dice que hay "efectos apalancamiento" cuando las "malas" noticias tienen un impacto mayor que las "buenas" sobre la volatilidad de los rendimientos. ${ }^{1}$

Existen diversos modelos teóricos y econométricos que explican el comportamiento de las series de rendimientos y de los inversionistas bursátiles. Los modelos teóricos más usados se sustentan en la teoría tradicional de portafolio (Markowitz, 1952; Tobin, 1958). En estos modelos suelen asumirse funciones de utilidad cuadráticas y distribuciones de densidad normales. Los modelos econométricos más usados para explicar el comportamiento de las

\footnotetext{
${ }^{1}$ Tradicionalmente los efectos apalancamiento se explican con argumentos de tipo contable y financiero. Particularmente Black (1976) señala que la disminución en el valor de la acción de una firma, atribuible a una "mala noticia", aumenta la razón contable de apalancamiento financiero de la misma. El mayor apalancamiento se traduce en mayores riesgos sobre la solvencia de la firma y sobre el valor de sus acciones. Los incrementos en dichos riesgos se reflejan en aumentos en la volatilidad de los precios de las acciones. Así, las "malas" noticias terminan teniendo un impacto mayor que las "buenas" noticias sobre la volatilidad de las series de precios de las acciones.
} 
series financieras son aquellos de la familia $\mathrm{ARCH} .{ }^{2}$ Estos modelos se caracterizan por tener una estructura dinámica autorregresiva en la varianza condicional de la variable en estudio por lo que se conocen como modelos de heteroscedasticidad condicional.

Los modelos teóricos y los econométricos presentan paralelismos y complementariedades entre sí: Los modelos teóricos enfatizan el análisis conjunto de la media y la varianza de los portafolios financieros. Los modelos econométricos enfatizan la modelación conjunta de la media y varianza de las series. De hecho, estos últimos se definen con base en las especificaciones de la media y la varianza condicionales y con base en la distribución de densidad que siguen las perturbaciones. En este contexto, no sobra señalar que ambos tipos de modelos suelen asumir que las variables de comportamiento tienen distribuciones de densidad simétricas (v.g. la distribución normal).

Los modelos teóricos y los econométricos asumen que los momentos de las distribuciones de densidad son necesarios para explicar las series de rendimientos bursátiles. Sin embargo, existen controversias acerca del número de momentos que debieran usarse para tomar decisiones óptimas. Particularmente la hipótesis tradicional de racionalidad indica que sólo es necesario conocer los dos primeros momentos (Tobin, 1958). ${ }^{3}$ La hipótesis de racionalidad de Samuelson indica que los inversionistas usan momentos superiores de las series bursátiles (Samuelson, 1970). Las controversias no son triviales, dado que las hipótesis tienen implicaciones sobre la rentabilidad y el manejo de riesgos bursátiles. ${ }^{4}$

\footnotetext{
${ }^{2}$ ARCH es acrónimo de "Autoregressive Conditional Heteroscedasticity".

${ }^{3}$ Esto ocurre porque en esta hipótesis se asume que las distribuciones de densidad son normales o porque las funciones de utilidad de los inversionistas son cuadráticas. Véase Tobin (1958).

${ }^{4}$ Adviértase que asumir simetría en la función de los rendimientos equivale a subestimar (sobreestimar) las probabilidades de rendimientos bajos cuando la distribución de los rendimientos es asimétrica a la derecha (izquierda). Véase Xiong y Idzorek (2011) para una descripción de los efectos de las asimetrías en los
} 
En esta investigación se propone un modelo TGARCH (Threshold-GARCH) con una distribución t de Student asimétrica para las perturbaciones ("shocks informacionales"). El mismo se construye usando la metodología de Fernández y Steel (1998) y el modelo TGARCH tradicional desarrollado por Zakoian (1994). ${ }^{5}$ El modelo se caracteriza por tener efectos apalancamiento y perturbaciones que siguen una distribución de densidad asimétrica. El mismo se usa para modelar series bursátiles y para evaluar la validez de las hipótesis de racionalidad en Latinoamérica. Las series analizadas son las de los rendimientos semanales de los índices accionarios de Argentina, Brasil, Chile, Colombia, México y Perú entre el 30 de diciembre de 2005 y el 6 de septiembre de 2013.

Metodológicamente la investigación se desarrolla en cuatro etapas. En la primera etapa se construye la distribución de densidad asimétrica que siguen las perturbaciones con base en la metodología de Fernández y Steel (1998). En la segunda, se introducen en el modelo efectos apalancamiento con base en la estructura dinámica propuesta por Zakoian (1994). El modelo propuesto se denomina TGARCH con distribución de densidad t de Student asimétrica. En la tercera etapa, se modelan las series de rendimientos bursátiles con los modelos TGARCH tradicional y propuesto. En la cuarta se evalúa la validez del modelo TGARCH propuesto y la pertinencia de las hipótesis de racionalidad.

Los resultados empíricos del estudio sugieren que: 1) Cada una de las series de rendimientos bursátiles analizadas puede describirse adecuadamente con el modelo

portafolios financieros.

${ }^{5}$ El nombre TGARCH se deriva de las principales características del modelo. TGARCH es acrónimo de "Threshold Generalized Autoregressive Conditional Heteroscedasticity". 
TGARCH propuesto; 2) la hipótesis de racionalidad de Samuelson es consistente con la evidencia de los mercados de Argentina, Brasil, Chile, Colombia y México; 3) hay efectos apalancamiento en las series de rendimientos de dichos mercados; 4) la hipótesis tradicional de racionalidad es consistente con la evidencia de Perú; y 5) las volatilidades estimadas mediante el modelo propuesto son mayores que las estimadas mediante el modelo TGARCH tradicional durante el periodo 2008-2009.

El estudio está organizado en siete secciones. La Sección 2 incluye la revisión de la literatura econométrica y de las hipótesis de racionalidad de los inversionistas que justifican el modelo. En la Sección 3 se construye la distribución de densidad t de Student asimétrica que define el comportamiento de las perturbaciones. En la sección 4 se desarrolla el modelo TGARCH con distribución de densidad t de Student asimétrica. En la Sección 5 se expone la metodología de evaluación del modelo propuesto y, en particular, de la hipótesis de Samuelson. En la Sección 6 se desarrolla el análisis econométrico. En la última sección, se sintetizan y discuten los resultados.

\section{Revisión de la literatura}

La modelación econométrica de series financieras ha tenido gran desarrollo en las últimas décadas. Según Engle (2004), los trabajos teóricos de Markowitz (1952) y Tobin (1958) sobre la racionalidad de los inversionistas financieros impulsaron el desarrollo de los modelos de la familia ARCH. La relevancia de estos modelos es que permiten describir y analizar series con comportamientos muy volátiles, curtosis excesivas, clusters de volatilidad, volatilidades no constantes y distribuciones no normales. Por esta razón, los 
modelos de esta familia se han convertido en la referencia inmediata para el análisis econométrico de series económicas y financieras. ${ }^{6}$

Los modelos de la familia ARCH se integran por especificaciones de la media y la varianza condicional y por supuestos sobre la distribución de densidad de las perturbaciones. Cabe señalar que las especificaciones de la varianza condicional son importantes porque los modelos se definen con base en las mismas. Estas especificaciones son postuladas porque la "varianza verdadera" de las series financieras no es observable. Por tanto, no sobra señalar que las especificaciones funcionales y los supuestos sobre las perturbaciones son relativamente subjetivos. Esta situación, en la práctica, conlleva a que haya controversias en torno a los supuestos de las especificaciones y la distribución de densidad de los modelos.

Existen modelos de la familia ARCH cuyas especificaciones de la varianza condicional asumen "shocks informacionales diferenciados". Entre estos modelos se encuentran el EGARCH y el TGARCH planteados, respectivamente, por Nelson (1991) y Zakoian (1994). Entre los trabajos que han usado estos modelos para estudiar la existencia de efectos apalancamiento en series financieras se encuentran los de Johnston y Soriano (2003) y Lorenzo-Valdés y Ruiz-Porras (2012). Particularmente, el primer trabajo estudia la volatilidad en varios mercados bursátiles durante la crisis asiática. El segundo modela los rendimientos bursátiles de México a nivel agregado y desagregado.

\footnotetext{
${ }^{6}$ Debe reconocerse que existen críticas hacia el uso de modelos ARCH para modelar series de tiempo. Un estudio reciente que critica estos modelos es el de Bonilla y Sepúlveda, (2011). Este trabajo es interesante porque critica el uso de los modelos $\mathrm{ARCH}$ para describir el comportamiento de los rendimientos bursátiles de economías emergentes.
} 
La teoría financiera usa varios supuestos estadísticos sobre comportamiento de los inversionistas para explicar la dinámica de las series financieras. La teoría de portafolios asume que los retornos bursátiles se distribuyen normalmente y que las funciones de utilidad de los inversionistas son cuadráticas (Tobin, 1958; Sharpe, 1964; Tsiang, 1972). Estos supuestos conllevan a que las decisiones de selección de portafolios sólo se expliquen en términos de la media y varianza de las series de rendimientos (Chunhachinda, et. al., 1997). Así, se postula que los inversionistas únicamente requieren conocer los dos primeros momentos de las distribuciones de densidad de los rendimientos para tomar decisiones óptimas.

La adopción de estos supuestos estadísticos ha generado controversias teóricas sobre la racionalidad que explica el comportamiento de los inversionistas bursátiles. La hipótesis tradicional de racionalidad indica que solo es necesario conocer los dos primeros momentos de las distribuciones de densidad de las series de rendimientos. Si bien la teoría financiera contemporánea se sustenta en el análisis de media-varianza; ${ }^{7}$ hay quienes argumentan que la hipótesis tradicional es insuficiente para explicar las decisiones de inversión. ${ }^{8}$ Entre los primeros estudios teóricos que propusieron alternativas a la hipótesis tradicional se encuentran los de Samuelson (1970), Jean (1971) y Arditti y Levi (1975).

La hipótesis de racionalidad de Samuelson indica que los inversionistas usan momentos superiores de las distribuciones de densidad para tomar sus decisiones. Particularmente Samuelson (1970) muestra que si las decisiones de inversión están restringidas en un

\footnotetext{
${ }^{7}$ Véase Elton et. al. (2013) para una revisión reciente y extensa de los desarrollos de la teoría moderna de portafolios e inversión.

${ }^{8}$ Las limitaciones de la hipótesis tradicional fueron formuladas inicialmente por Borch (1969) y Feldstein (1969).
} 
intervalo de tiempo finito, asumiendo funciones cuadráticas de utilidad, la media y varianza se vuelven insuficientes para explicar el comportamiento de los inversionistas. De hecho, él indica que la teoría tradicional solo puede ofrecer una aproximación del comportamiento de los inversionistas “cuando el riesgo es muy limitado" (Samuelson, 1970:542).

Econométricamente la pertinencia de las hipótesis mencionadas puede evaluarse en términos de los supuestos sobre las distribuciones de densidad. Particularmente, la hipótesis de Samuelson puede evaluarse analizando la validez de los momentos superiores para describir los rendimientos. Sin embargo, esta validación no es común en la modelación econométrica. Los modelos ARCH suelen asumir que las perturbaciones estandarizadas siguen distribuciones de densidad donde el tercer momento es cero. Incluso, en muchos estudios se suele usar directamente la distribución normal. Así, los análisis empíricos postulan, sin cuestionar, la validez de la hipótesis tradicional.

Existen algunos modelos teóricos de la familia ARCH que usan momentos superiores a la media y varianza. Estos modelos usan expresiones dinámicas para definir momentos condicionales de las distribuciones de probabilidad. Esto significa que los momentos presentes se explican con base en los rezagos de los momentos y de las perturbaciones. Particularmente Hansen (1994) es quien primero construye un modelo con una distribución $t$ de Student asimétrica. Harvey y Siddique (1999) y (2000) extienden ese trabajo para estimar coeficientes de asimetría. ${ }^{9}$ Más recientemente, Durán-Vázquez, Lorenzo-Valdés y Ruíz-Porras (2013) construyen un modelo con una distribución normal asimétrica.

\footnotetext{
${ }^{9}$ El coeficiente de asimetría es una medida del sesgo de la distribución de la probabilidad de una serie de tiempo. Se le define como el tercer momento central estandarizado. Si el sesgo es negativo, la función de probabilidad es asimétrica a la izquierda (i.e. la probabilidad de tener rendimientos relativamente altos es mayor que la de tener rendimientos bajos). Si el sesgo es positivo, la función es asimétrica a la derecha.
} 
En la literatura son escasos los análisis que usan modelos $\mathrm{ARCH}$ que consideren momentos superiores a la varianza. Entre estos estudios destaca el de Chunhachinda, et. al. (1997). Ellos muestran que los inversionistas suelen cambiar sus elecciones de portafolio cuando suponen sesgos en los mercados. Chen, Hong y Stein (2001), por su parte, hallan evidencia de que los modelos con asimetría condicional son capaces de predecir "cracs" bursátiles usando estimaciones de los sesgos de los rendimientos financieros. Prakash, Chang y Pactwa, (2003) y Canela y Pedreira-Collazo, (2007) describen los rendimientos bursátiles de varios mercados emergentes mediante modelos asimétricos.

Los modelos señalados tienen similitudes y diferencias con el propuesto aquí. Todos los modelos tienen una estructura dinámica de tipo ARCH para describir el comportamiento de las series financieras. Asimismo, en ellos se asume que las perturbaciones siguen distribuciones de densidad asimétricas. Las diferencias se refieren a la naturaleza de la asimetría de las distribuciones y a los impactos que tienen las perturbaciones rezagadas sobre la volatilidad. Particularmente el modelo TGARCH propuesto asume que el tercer momento es un parámetro que no está condicionado temporalmente. Asimismo, asume que las series pueden experimentar efectos apalancamiento tal como la hace Zakoian (1994).

Finalmente, no sobra enfatizar que la modelación de las series bursátiles y el análisis del comportamiento de los inversionistas resultan de gran interés teórico y empírico. En el ámbito financiero, la modelación de series es relevante para administrar riesgos, seleccionar portafolios y valuar instrumentos. En este contexto, el análisis de las hipótesis de racionalidad es importante para evaluar la rentabilidad y los riesgos de manera adecuada. 
Además, la estimación de los efectos de "shocks informacionales" resulta importante para evaluar los impactos de las noticias en las economías emergentes. De hecho, con base en estas consideraciones se justifican los análisis desarrollados en las siguientes secciones.

\section{La función de distribución $t$ asimétrica}

En esta sección se define la función de distribución de densidad asimétrica usada describir el comportamiento de las perturbaciones $u$ del modelo TGARCH propuesto aquí. Esta función de probabilidad asimétrica se construye siguiendo la metodología de Fernández y Steel (1998). La relevancia de esta metodología es que permite transformar distribuciones simétricas en asimétricas de una manera muy sencilla. La misma solamente requiere usar un escalar $\lambda$, i.e. un parámetro de asimetría, para hacer dicha transformación. Particularmente aquí se usa la misma para transformar una distribución de densidad t de Student en una distribución "t de Student asimétrica". ${ }^{10}$

Matemáticamente, se plantea la transformación propuesta considerando la función de densidad $\mathrm{t}$ de Student con media cero, varianza uno y grados de libertad $v$, donde $v>2$.

Esta función de densidad para la variable $x_{t}, x_{t} \sim t(0,1, v)$, se define como:

$$
f\left(x_{t}\right)=\frac{\Gamma\left(\frac{v+1}{2}\right)}{\Gamma\left(\frac{v}{2}\right)} \frac{1}{\sqrt{\pi(v-2)}}\left[1+\frac{x_{t}^{2}}{v-2}\right]^{-\left(\frac{v+1}{2}\right)}
$$

La función logaritmo de verosimilitud asociada a la función (1) es:

\footnotetext{
${ }^{10}$ Durán Vázquez, Lorenzo Valdés y Ruiz Porras (2013) usan esta metodología para construir una "normal asimétrica".
} 
$L(\theta)=\ln \left[\Gamma\left(\frac{v+1}{2}\right)\right]-\ln \left[\Gamma\left(\frac{v}{2}\right)\right]-0.5 \ln [\pi(v-2)]-0.5 \sum_{t=1}^{T}\left[\ln \sigma_{t}^{2}+(1+v) \ln \left(1+\frac{x_{t}^{2}}{v-2}\right)\right]$

donde $\theta$ es el conjunto de parámetros a estimar y $\Gamma(\bullet)$ es la función gama.

La metodología propuesta introduce asimetría en las distribuciones de densidad mediante factores de escala inversos en los valores positivos y negativos de $x_{t}$. Estos factores de definen con el escalar $\lambda>0$ (parámetro de asimetría). Particularmente, si este escalar es fijo, la función de densidad para la variable $x_{t}$ que se distribuye siguiendo una distribución de densidad $t$ de Student asimétrica queda definida como:

$$
f\left(x_{t} \mid \lambda\right)=\frac{2}{(\lambda+1 / \lambda)}\left\{f\left(\frac{x_{t}}{\lambda}\right) I_{[0, \infty)}\left(x_{t}\right)+f\left(\lambda x_{t}\right) I_{(-\infty, 0)}\left(x_{t}\right)\right\},
$$

donde $I_{A}=\left\{\begin{array}{ll}1 & \text { si sucede } A \\ 0 & \text { no sucede } A\end{array}\right.$.

La función (3) generaliza la distribución de densidad t de Student con base en el parámetro de asimetría $\lambda$ (escalar). Si $\lambda=1$, la función resultante es la distribución de densidad $\mathrm{t}$ de Student simétrica. Si $\lambda \neq 1$, la función resultante es la distribución de densidad t de Student asimétrica. Particularmente, si $\lambda<1$, la función es una distribución sesgada a la izquierda y si $\lambda>1$, la función es una distribución sesgada a la derecha. Por tanto, el sesgo de la función (3) depende de los valores de $\lambda$. En este contexto, puede generalizarse la notación definida anteriormente: Si la variable $x_{t}$, se distribuye siguiendo una $t$ de Student asimétrica y estandarizada, puede denotarse dicha situación como $x_{t} \sim t(0,1, \lambda, v)$. 
Fernández y Steel (1998) y Lambert y Laurent (2001) muestran que la función logaritmo de

verosimilitud para una variable $z_{t}$, definida como $z_{t}=\frac{u_{t}-m}{s}$, que se distribuye siguiendo una $t$ de Student asimétrica y estandarizada, $z_{t} \sim t(0,1, \lambda, v)$, es:

$$
\begin{aligned}
L(\theta)= & \ln \left[\Gamma\left(\frac{v+1}{2}\right)\right]-\ln \left[\Gamma\left(\frac{v}{2}\right)\right]-0.5 \ln [\pi(v-2)]+\ln \left(\frac{2}{\lambda+\frac{1}{\lambda}}\right)+\ln (s) \\
& -0.5 \sum_{t=1}^{T}\left[\ln \sigma_{t}^{2}+(1+v) \ln \left(1+\frac{s z_{t}+m}{v-2} \lambda^{-I_{t}}\right)\right]
\end{aligned}
$$

donde $m$ y $s$ son la media y desviación estándar de las perturbaciones, $u,{ }^{11}$; y donde la

función indicadora, $I_{t}$, queda definida como $I_{t}=\left\{\begin{array}{ll}1 & \text { si } z_{t} \geq-\frac{m}{s} \\ -1 & \text { si } z_{t}<-\frac{m}{s}\end{array}\right.$.

La función de densidad $t$ de Student asimétrica, $z_{t} \sim t(0,1, \lambda, v)$, provee un marco para evaluar las diversas hipótesis de racionalidad de los inversionistas bursátiles. La hipótesis tradicional, asociada a la teoría de portafolio, supone que $\lambda=1$ (i.e. las probabilidades de tener rendimientos altos y bajos son iguales). La hipótesis de Samuelson supone que $\lambda<1$ (i.e. la probabilidad de tener rendimientos relativamente altos es mayor que la de tener

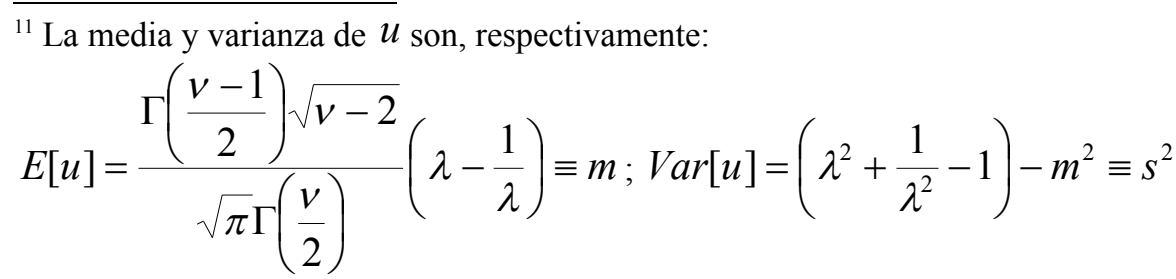


rendimientos bajos). En este contexto, debe señalarse que en este estudio no se postulan "a priori" los valores del coeficiente de asimetría $\lambda$. Se estiman con base en el comportamiento dinámico de las series de tiempo analizadas.

\section{Modelo TGARCH con distribución de densidad t de Student asimétrica}

En esta sección se desarrolla el modelo TGARCH (Threshold GARCH) con distribución de densidad t de Student asimétrica. El modelo TGARCH propuesto se construye con base en el desarrollado originalmente por Zakoian (1994) y los desarrollos matemáticos de la sección previa. El objetivo del modelo propuesto consiste en describir la dinámica temporal de las series de rendimientos representativos, $r_{i t}$, de los mercados latinoamericanos (denotados por el subíndice $i$ ). Para describir dicha dinámica, como ya se ha mencionado, se asume que la media y varianza de los rendimientos siguen una estructura de tipo TGARCH. Asimismo se asume que las perturbaciones $u_{i t}$ siguen una distribución t de Student asimétrica.

El modelo TGARCH tradicional describe la dinámica de una serie de tiempo con base en suponer efectos apalancamiento en la especificación de la varianza y distribuciones de densidad simétricas. Particularmente, Zakoian (1994) plantea que las perturbaciones se comportan siguiendo una función de densidad simétrica y estandarizada (v.g. normal, t de Student tradicionales). En el ámbito financiero este supuesto se justifica bajo la hipótesis de que los inversionistas toman sus decisiones de portafolio considerando únicamente la media y las varianza de los rendimientos de los activos. Por tanto, en el modelo TGARCH tradicional se asume que el tercer momento de la distribución de densidad es cero. 
Empíricamente, el modelo TGARCH se usa para describir los efectos en la volatilidad de las series de tiempo cuando hay "shocks informacionales" cualitativamente diferenciados. Para ello, la especificación de la varianza propuesta por Zakoian (1994) permite que la desviación estándar condicionada de las series, $\sigma_{i t}$, dependa del signo de los shocks rezagados $u_{i t-1}$ como se muestra en la ecuación (4). Particularmente, la especificación de la varianza permite que el impacto de shocks positivos sea $\alpha_{i 1}$; mientras que, el impacto de los negativos sea $\alpha_{i 1}+\gamma_{i}$. En el contexto de esta investigación, no sobra señalar que sí $\gamma_{i}>0$, las series de rendimientos bursátiles reflejan shocks informacionales conocidos como efectos apalancamiento.

Econométricamente el modelo propuesto aquí puede describirse como de tipo TGARCH con una distribución $t$ de Student asimétrica y estandarizada. Particularmente, el modelo se plantea con base en una estructura dinámica, $\mathrm{AR}(1)-\mathrm{TGARCH}(1,1)$, definida como:

$$
\begin{aligned}
& r_{i t}=\phi_{i 0}+\phi_{i 1} r_{i t-1}+u_{i t} \\
& u_{i t}=\sigma_{i t} z_{i t} \\
& \sigma_{i t}^{2}=\alpha_{i 0}+\alpha_{i l} u_{i t-1}^{2}+\gamma_{i} u_{i t-1}^{2} I_{i t-1}+\beta_{i} \sigma_{i t-1}^{2} .
\end{aligned}
$$

donde $I_{i t}=\left\{\begin{array}{ll}1 & u_{i t-1}<0 \\ 0 & u_{i t-1} \geq 0\end{array}\right.$.

El modelo TGARCH propuesto está integrado por tres expresiones que definen la estructura dinámica de las series y la distribución de densidad de probabilidad de las perturbaciones. La primera expresión es la especificación de la media de los rendimientos. 
La segunda es la condición que define un proceso ARCH (véase Bollerslev 2010). La tercera es la especificación de la varianza condicional propuesta por Zakoian (1994). Esta especificación permite describir capturar los efectos en la volatilidad asociados a "shocks informacionales" diferenciados. La distribución de densidad se postula como una t de Student asimétrica y estandarizada.

\section{Metodología de análisis}

La investigación empírica se sustenta en el análisis de seis series representativas de los rendimientos de los mercados bursátiles latinoamericanos. Los mercados estudiados son los de Argentina, Brasil, Chile, Colombia, México y Perú. Las series de los rendimientos se construyen usando los valores de cierre de los índices representativos de precios de cada país $i$ durante el periodo semanal $t, P_{i t}$. Asimismo, se asume que los índices de precios siguen un proceso continuo de interés compuesto. Por tanto, cada observación de la serie de rendimientos semanales de un país $i$ en el periodo $t, r_{i t}$, se construye de la siguiente manera:

$$
r_{i t}=\ln P_{i t}-\ln P_{i t-1}
$$

La validez de modelo TGARCH con una distribución $\mathrm{t}$ de Student asimétrica y la pertinencia de las hipótesis de racionalidad se evalúan en tres etapas. En la primera, se modelan los comportamientos de las series de los rendimientos representativos de los

mercados bursátiles analizados. En la segunda, se investiga si existen "shocks informacionales diferenciados" en dichos mercados. En la tercera, se explora si se cumple la hipótesis de racionalidad de Samuelson en los mismos. Los análisis desarrollados en cada 
etapa se sustentan en estimaciones de los modelos TGARCH tradicional y propuesto para cada serie de rendimientos bursátiles para facilitar el análisis comparativo y estadístico.

Econométricamente, las estimaciones realizadas para cada serie mediante los modelos TGARCH tradicional y propuesto son muy similares. En todos los casos, la técnica de estimación usada es la de máxima verosimilitud (Maximum Likelihood). Estructuralmente, en ambos modelos se asume que las series siguen un comportamiento dinámico de tipo AR(1)-TGARCH(1,1,). Por esta razón, las estimaciones de ambos modelos refieren a parámetros similares. Los parámetros estimados incluyen a aquellos relativos a las especificaciones de la media $\left(\phi_{i 0}, \phi_{i 1}\right)$, de la varianza condicional $\left(\alpha_{i 0}, \alpha_{i 1}, \gamma_{i}, \beta_{i}\right)$ y a los grados de libertad $v_{i}$.

Estadísticamente, la única diferencia en las estimaciones realizadas mediante ambos modelos se refiere a la naturaleza de las distribuciones de densidad de las perturbaciones. El modelo TGARCH tradicional asume que las perturbaciones siguen una distribución $\mathrm{t}$ de Student simétrica. El modelo TGARCH propuesto asume que las perturbaciones siguen una distribución $\mathrm{t}$ de Student asimétrica. Por esta razón, en este último modelo, adicionalmente se estima el parámetro $\lambda_{i}$ con base en el trabajo de Lambert y Laurent (2001). La estimación de este parámetro se realiza asumiendo la existencia de una solución estacionaria para la especificación de la varianza condicional. ${ }^{12}$

${ }^{12}$ Ding, Granger y Engle (1993) muestran la solución estacionaria para un modelo APARCH (Asymmetric Power GARCH) es también aplicable para el modelo TGARCH. Esta solución está definida como:

$$
E\left(\sigma_{i t}^{\delta}\right)=\frac{\alpha_{i 0}}{1-\alpha_{i 1} E(|z|-\gamma z) \delta-\beta_{i 1}} .
$$


Metodológicamente, el análisis de las estimaciones obtenidas se hace mediante estadísticos de bondad de ajuste y pruebas de significancia. Aquí se utilizan los estimadores de bondad de ajuste del logaritmo de verosimilitud (Log-Likelihood) y del criterio de información de Akaike $(A I C)$. Se usan ambos estimadores porque consideran criterios alternativos de bondad de ajuste. El criterio de decisión se sustenta en comparar las estimaciones, medidas en valor absoluto, de los estimadores asociados a cada modelo. Particularmente si el modelo TGARCH propuesto describiera mejor las series, ocurriría que las estimaciones serían mayores que las correspondientes al del modelo TGARCH tradicional.

La existencia de "shocks informacionales diferenciados" y la pertinencia de las hipótesis se evalúan mediante pruebas de significancia individual de coeficientes. La significancia de cada parámetro estimado se evalúa usando los p-values asociados a los estadísticos z de significancia individual. Particularmente, la validación de los efectos apalancamiento ocurriría si el parámetro estimado de $\gamma_{i}$ fuera significativo y positivo. Si esto no fuera así, no se podría sustentar su existencia. La hipótesis de Samuelson se podría validar si el parámetro $\lambda_{i}$ fuera significativo y de magnitud menor a uno. Si $\lambda_{i}$ fuera unitario se podría validar la hipótesis tradicional de portafolio.

Esta solución se obtiene considerando una especificación de la varianza condicional de tipo APARCH dada de la manera siguiente:

$$
\sigma_{i t}^{\delta}=\alpha_{0}+\alpha_{1}\left(\left|u_{t-1}\right|-\gamma_{i} u_{t-1}\right)+\beta_{i 1} \sigma_{i t-1}^{\delta}
$$

Particularmente, si se asume una distribución t de Student asimétrica, Lambert y Laurent (2001) muestran que

$$
E(|z|-\gamma z)^{\partial}=\left\{\lambda^{-(1+\delta)}(1+\gamma)^{\delta}+\lambda^{(1+\delta)}(1-\gamma)^{\delta}\right\} \frac{\Gamma\left(\frac{\delta+1}{2}\right) \Gamma\left(\frac{v-\delta}{2}\right)(v-2)^{\frac{1+\delta}{2}}}{\left(\lambda+\frac{1}{\lambda}\right) \sqrt{(v-2) \pi \Gamma\left(\frac{v}{2}\right)}}
$$


Finalmente, no sobra enfatizar que la validación empírica del modelo TGARCH propuesto tiene implicaciones en la dinámica de los mercados bursátiles latinoamericanos. La constatación de efectos apalancamiento implicaría que la información tiene impactos cualitativa y cuantitativamente diferenciados sobre la volatilidad presente de las series bursátiles. La validación de la hipótesis de Samuelson implicaría que el comportamiento de los inversionistas es más complejo que el supuesto por los modelos de Markowitz (1952) y Tobin (1958). Más aun, implicaría que la hipótesis tradicional de racionalidad sería insuficiente para evaluar la rentabilidad y los riesgos bursátiles de manera adecuada.

\section{Análisis econométrico}

Este estudio usa la base de datos de Economática para obtener una muestra de datos bursátiles representativos de los mercados latinoamericanos. La muestra incluye los valores de cierre semanal de las principales índices de precios representativos de Argentina, Brasil, Chile, Colombia, México y Perú registrados entre el 30 de diciembre de 2005 y el 6 de septiembre de 2013. Así la muestra se integra por 6 series de índices de precios donde cada serie incluye 402 observaciones semanales. Esta muestra nos sirve para construir seis series de rendimientos bursátiles con base en la ecuación (5). Estas series de rendimientos, cada una con 401 observaciones, constituyen la base de datos de esta investigación.

Estadísticamente el uso de modelos de la familia ARCH se puede justificar con base en ciertas características de las series de tiempo. Estas características incluyen curtosis altas, clusters de volatilidad, distribuciones no normales, coeficientes de asimetría diferentes de cero y movimientos conjuntos de la media y volatilidad entre los distintos mercados. 
Particularmente si las series muestran coeficientes de asimetrías diferentes de cero puede justificarse la necesidad de considerar distribuciones de densidad asimétricas para describir su comportamiento. Estas características se pueden detectar mediante estadísticas descriptivas. La Tabla 1 muestra dichas estadísticas para las series analizadas.

\begin{tabular}{|l|c|c|c|c|c|c|}
\hline \multicolumn{1}{|c|}{ País } & Media & Desv. Est. & Coef. Asim. & Curtosis & Jarque-Bera & P-value \\
\hline Argentina & 0.0025 & 0.0455 & -1.0216 & 11.2646 & 1210.99 & 0.000 \\
\hline Brasil & 0.0011 & 0.0382 & -0.5321 & 7.6940 & 387.08 & 0.000 \\
\hline Chile & 0.0016 & 0.0280 & -1.2654 & 13.9494 & 2110.17 & 0.000 \\
\hline Colombia & 0.0009 & 0.0315 & -1.7968 & 12.9144 & 1858.11 & 0.000 \\
\hline México & 0.0020 & 0.0330 & -0.1661 & 8.8052 & 564.93 & 0.000 \\
\hline Perú & 0.0031 & 0.0444 & -1.1564 & 14.0829 & 2141.67 & 0.000 \\
\hline
\end{tabular}

Tabla 1. Estadísticas descriptivas de los rendimientos y prueba de normalidad de Jarque-Bera.

La Tabla 1 muestra la estadística descriptiva de las series bursátiles. La misma está integrada por seis columnas. La primera columna muestra el rendimiento semanal promedio. La segunda muestra la desviación estándar de los rendimientos diarios que es estimador estático de la volatilidad. La tercera columna muestra el coeficiente de asimetría, también conocido como sesgo. La cuarta columna muestra la curtosis o ancho de colas. La quinta columna muestra el estimador Jarque-Bera. La sexta columna muestra el p-value asociado a dicho estimador. La hipótesis nula asociada a los estimadores Jarque-Bera es que los rendimientos se distribuyen normalmente.

La Tabla 1 muestra que todas las series de rendimientos bursátiles latinoamericanos tienen curtosis leptocúrticas, y que sus distribuciones son sesgadas (asimétricas). En todas las series las curtosis estimadas son mayores a 3 y los coeficientes de asimetría registran 
valores negativos. Más aun, en todos los casos las pruebas Jarque-Bera rechazan la hipótesis nula de normalidad. Analíticamente debe destacarse que los sesgos negativos indican que las probabilidades de obtener rendimientos altos son mayores que aquellas de obtener bajos rendimientos. Por tanto, el análisis, en principio, justifica la conveniencia de usar distribuciones asimétricas para describir los rendimientos.

Los modelos TGARCH tradicional y propuesto comparten una estructura similar para efectos de estimación econométrica. En ambos modelos, las especificaciones requieren estimar los parámetros de las medias $\phi_{i 0} \mathrm{y} \phi_{i 1}$; de las varianzas $\alpha_{i 0}, \alpha_{i 1}, \gamma_{i}$ y $\beta_{i}$; y de los grados de libertad $v_{i}$. En añadidura, las estimaciones del modelo TGARCH propuesto incluyen al parámetro de asimetría $\lambda_{i}$. Como ya se ha indicado, los rendimientos bursátiles representativos de cada mercado latinoamericano se describen usando ambos modelos por comparabilidad. Estas estimaciones están sintetizadas en las Tablas 2 y 3. El análisis de la magnitud del parámetro $\lambda_{i}$ se desarrolla en la Tabla 4.

La Tabla 2 muestra las estimaciones del modelo AR(1)-TGARCH(1,1) asumiendo que las perturbaciones se distribuyen con una distribución $\mathrm{t}$ de Student asimétrica. Estas estimaciones del modelo TGARCH tradicional se sintetizan en seis columnas. Cada columna se refiere a las estimaciones relativas a un mercado determinado. Los primeros dos pares de filas se refieren a los parámetros estimados $\phi_{i 0}$ y $\phi_{i 1}$, respectivamente. Los siguientes cinco pares se refieren a los parámetros $\alpha_{i 0}, \alpha_{i 1}, \gamma_{i}, \beta_{i} \mathrm{y} v_{i}$. En cada par, la fila 
superior muestra los coeficientes estimados y la inferior muestra los p-values de los estadísticos z de significancia individual de los parámetros estimados.

\begin{tabular}{|c|c|c|c|c|c|c|c|}
\hline & & Argentina & Brasil & Chile & Colombia & México & Perú \\
\hline \multirow[t]{2}{*}{$\phi_{0}$} & Coef. & 0.0036 & 0.0010 & 0.0028 & 0.0031 & 0.0026 & 0.0034 \\
\hline & Prob. & 0.0454 & 0.5436 & 0.0095 & 0.0075 & 0.0402 & 0.0547 \\
\hline \multirow[t]{2}{*}{$\phi_{1}$} & Coef. & -0.0085 & -0.0376 & 0.0474 & -0.0724 & -0.0789 & 0.1278 \\
\hline & Prob. & 0.8718 & 0.5180 & 0.3732 & 0.0866 & 0.1411 & 0.0187 \\
\hline \multirow[t]{2}{*}{$\alpha_{0}$} & Coef. & 0.0001 & 0.0001 & 0.0001 & 0.0000 & 0.0000 & 0.0002 \\
\hline & Prob. & 0.0315 & 0.0096 & 0.0056 & 0.0000 & 0.0706 & 0.0284 \\
\hline \multirow[t]{2}{*}{$\alpha_{1}$} & Coef. & 0.0478 & -0.0350 & 0.0254 & -0.0980 & 0.0106 & 0.1893 \\
\hline & Prob. & 0.3623 & 0.3951 & 0.7261 & 0.0001 & 0.8075 & 0.0456 \\
\hline \multirow[t]{2}{*}{$\gamma$} & Coef. & 0.1066 & 0.1656 & 0.3465 & 0.1161 & 0.1774 & 0.0195 \\
\hline & Prob. & 0.0590 & 0.0002 & 0.0084 & 0.0000 & 0.0023 & 0.8452 \\
\hline \multirow[t]{2}{*}{$\beta$} & Coef. & 0.8156 & 0.8831 & 0.6132 & 0.9884 & 0.8596 & 0.6643 \\
\hline & Prob. & 0.0000 & 0.0000 & 0.0000 & 0.0000 & 0.0000 & 0.0000 \\
\hline \multirow[t]{4}{*}{$v$} & Coef. & 5.8626 & 15.5709 & 5.3362 & 3.9383 & 7.4372 & 5.5756 \\
\hline & Prob. & 0.0021 & 0.2013 & 0.0002 & 0.0000 & 0.0032 & 0.0001 \\
\hline & Logl & 736.51 & 791.48 & 940.62 & 895.47 & 869.78 & 746.79 \\
\hline & Akaike & -3.64754 & -3.92239 & -4.66811 & -4.44236 & -4.31392 & -3.69895 \\
\hline
\end{tabular}

Tabla 2. Estimaciones de las series de rendimientos bursátiles con base en el modelo AR(1)-TGARCH(1,1) con una distribución $\mathrm{t}$ de Student simétrica.

La Tabla 2 muestra que hay efectos apalancamiento significativos en casi todas las series latinoamericanas de rendimientos bursátiles. La excepción se refiere a la serie representativa del mercado peruano. Los parámetros estimados de las especificaciones de las varianzas son, en la mayoría de los casos, consistentes con los postulados teóricamente.

Los coeficientes estimados $\alpha_{i 0}, \alpha_{i 1}, \gamma_{i}$ y $\beta_{i}$, son significativos y positivos para un total de $6,1,5$ y 6 series, respectivamente. ${ }^{13}$ Asimismo, los coeficientes $\phi_{i 0}$ y $\phi_{i 1}$ son significativos y positivos para 5 y 1 series. Por tanto, la evidencia sugiere que los inversionistas bursátiles reaccionan de manera diferenciada ante las buenas y malas noticias.

\footnotetext{
${ }^{13}$ El nivel de significancia considerado es 0.10 .
} 


\begin{tabular}{|c|c|c|c|c|c|c|c|}
\hline & & Argentina & Brasil & Chile & Colombia & México & Perú \\
\hline \multirow{2}{*}{$\overline{\phi_{0}}$} & Coef. & 0.0021 & -0.0001 & 0.0033 & 0.0019 & 0.0011 & 0.0030 \\
\hline & Prob. & 0.2647 & 0.9272 & 0.0318 & 0.0263 & 0.3836 & 0.1152 \\
\hline \multirow{2}{*}{$\phi_{1}$} & Coef. & -0.0130 & -0.0566 & -0.0121 & -0.0654 & -0.1144 & 0.1321 \\
\hline & Prob. & 0.8036 & 0.3112 & 0.8420 & 0.0982 & 0.0242 & 0.0149 \\
\hline \multirow{2}{*}{$\alpha_{0}$} & Coef. & 0.0001 & 0.0000 & 0.0001 & 0.0000 & 0.0000 & 0.0002 \\
\hline & Prob. & 0.0260 & 0.0074 & 0.0067 & 0.0000 & 0.0943 & 0.0269 \\
\hline \multirow{2}{*}{$\alpha_{1}$} & Coef. & 0.0413 & -0.0189 & -0.0499 & -0.1142 & -0.0039 & 0.1864 \\
\hline & Prob. & 0.3694 & 0.6020 & 0.3647 & 0.0000 & 0.9088 & 0.0461 \\
\hline \multirow[t]{2}{*}{$\gamma$} & Coef. & 0.0887 & 0.1503 & 0.2467 & 0.1313 & 0.1558 & 0.0209 \\
\hline & Prob. & 0.0688 & 0.0002 & 0.0017 & 0.0000 & 0.0002 & 0.8398 \\
\hline \multirow[t]{2}{*}{$\beta$} & Coef. & 0.8571 & 0.9072 & 0.7112 & 1.0007 & 0.9121 & 0.6612 \\
\hline & Prob. & 0.0000 & 0.0000 & 0.0000 & 0.0000 & 0.0000 & 0.0000 \\
\hline \multirow[t]{2}{*}{$\lambda$} & Coef. & 0.8635 & 0.6918 & 0.8441 & 0.8588 & 0.7328 & 0.9692 \\
\hline & Prob. & 0.0000 & 0.0000 & 0.0000 & 0.0000 & 0.0000 & 0.0000 \\
\hline \multirow[t]{4}{*}{$v$} & Coef. & 6.0587 & 68.1710 & 9.8821 & 4.1449 & 11.5576 & 5.6723 \\
\hline & Prob. & 0.0027 & 0.7976 & 0.0006 & 0.0000 & 0.1040 & 0.0001 \\
\hline & Logl & 964.27 & 1025.51 & 1144.64 & 1126.95 & 1102.39 & 973.64 \\
\hline & Akaike & -4.79334 & -5.10032 & -5.69745 & -5.60875 & -5.48566 & -4.84030 \\
\hline
\end{tabular}

Tabla 3. Estimaciones de las series de rendimientos bursátiles con base en el modelo AR(1)-TGARCH(1,1) con una distribución $t$ de Student asimétrica.

La Tabla 3 muestra las estimaciones obtenidas mediante el modelo TGARCH con una distribución $\mathrm{t}$ de Student asimétrica. La tabla confirma que hay efectos apalancamiento significativos en las series de rendimientos de Argentina, Brasil, Chile, Colombia y México. Los signos y significancia estimados de los parámetros de las especificaciones de las varianzas son cualitativamente similares a los reportados en la Tabla 2. Más importante, las estimaciones de $\lambda_{i}$ resultan positivas y significativas en todos los casos. Por tanto la evidencia sugiere que los inversionistas consideran momentos superiores a la media y la varianza para tomar sus decisiones.

Las Tablas 2 y 3 permiten comparar la bondad de ajuste de los modelos TGARCH tradicional y propuesto. Como ya se mencionado, esta comparación se hace usando los estimadores Log-Likelihood y AIC. Particularmente, y como resultado del análisis 
comparativo, puede concluirse que los resultados validan la pertinencia del modelo TGARCH con una distribución $\mathrm{t}$ de Student asimétrica. De hecho, cada una de las series analizadas puede describirse relativamente mejor con el modelo TGARCH propuesto que con el modelo tradicional. Así, puede concluirse que el análisis justifica la pertinencia de asumir que las perturbaciones siguen distribuciones de probabilidad asimétricas.

La Tabla 4 muestran los resultados de analizar la validez de las hipótesis de racionalidad de los inversionistas en los mercados latinoamericanos. Este análisis se hace evaluando la hipótesis nula de que la distribución de densidad de los rendimientos es simétrica; contra la alternativa de que la distribución es sesgada a la izquierda. Como puede advertirse, la hipótesis nula se rechaza en casi todos los casos (la excepción se refiere a Perú). Por tanto se confirma que es preferible utilizar distribuciones asimétricas para describir el comportamiento de las perturbaciones. Asimismo, se muestra que la hipótesis de Samuelson es consistente con la evidencia de la mayoría de los mercados analizados.

\begin{tabular}{|c|c|c|c|c|c|c|c|}
\hline & & Argentina & Brasil & \multicolumn{1}{c}{ Chile } & Colombia & México & Perú \\
\hline$\lambda$ & Coef. & 0.8635 & 0.6918 & 0.8441 & 0.8588 & 0.7328 & 0.9692 \\
\hline & Error est. & 0.0641 & 0.0689 & 0.0707 & 0.0644 & 0.0741 & 0.0734 \\
\hline & Estadístico & -2.1278 & -4.4739 & -2.2062 & -2.1924 & -3.6081 & -0.4195 \\
\hline & Prob. & 0.01668 & 0.00000 & 0.01368 & 0.01418 & 0.00015 & 0.33742 \\
\hline
\end{tabular}

Tabla 4. Prueba de la pertinencia de la hipotesis de racionalidad de Samuelson. La hipotesis nula es que $\lambda$ unitario. La alternativa es que $\lambda$ es menor a uno.

Las Figuras 1 y 2 muestran los ejercicios de modelación realizados para cada mercado bursátil. Particularmente, la Figura 1 grafica las series de rendimientos y sus volatilidades ajustadas con base en el modelo tradicional. La Figura 2 grafica sus contrapartes ajustadas con base en el modelo propuesto. En este contexto, conviene destacar que las volatilidades 
estimadas mediante el modelo propuesto son ligeramente mayores que las estimadas mediante el modelo tradicional para el periodo 2008-2009. Estas diferencias ocurren en casi todas las series analizadas. ${ }^{14}$ Estas diferencias son importantes porque sugieren que suponer simetría en las distribuciones puede llevar a subestimar riesgos en los mercados financieros.

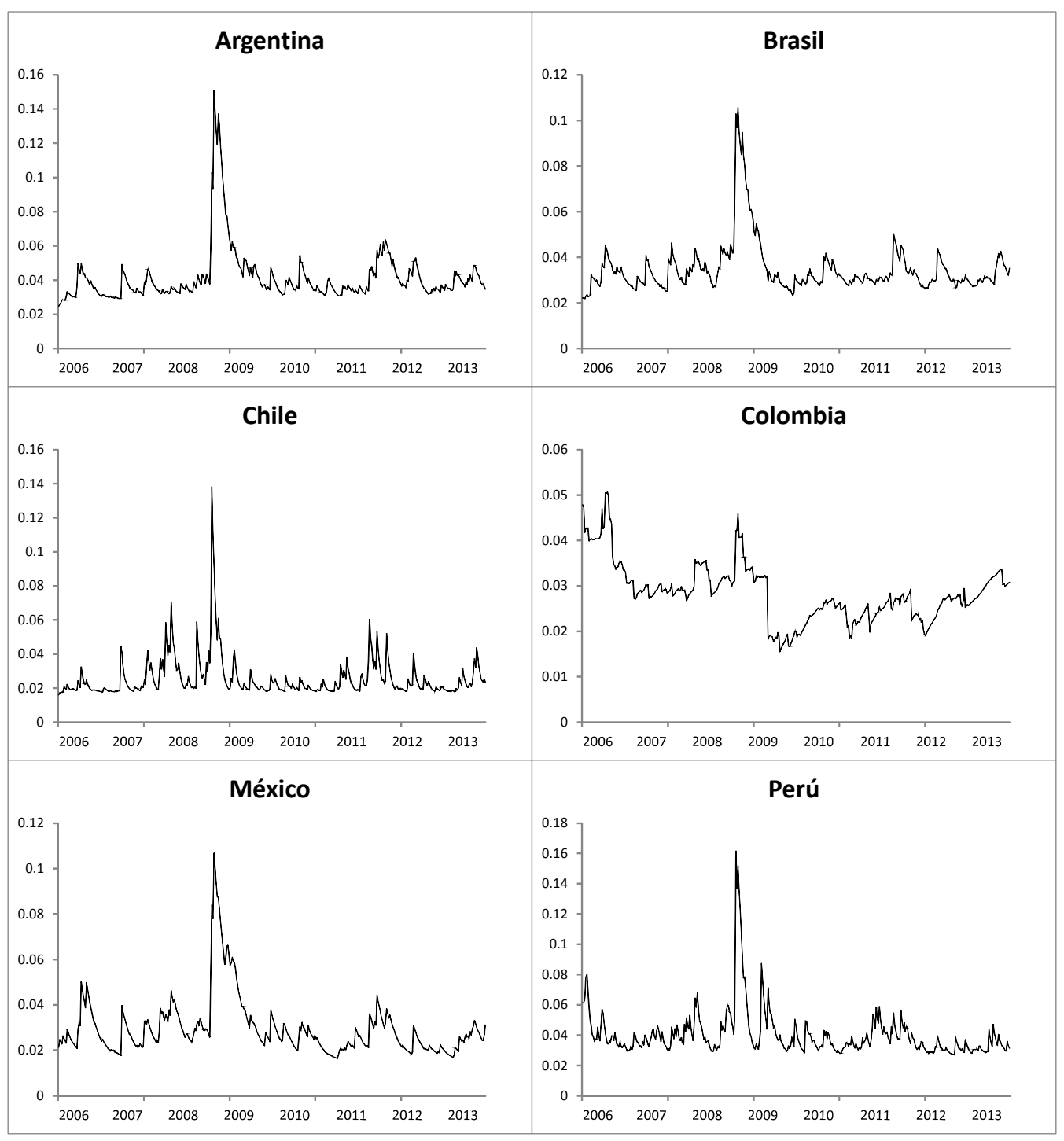

Figura 1. Gráficas de las volatilidades estimadas mediante el modelo TGARCH tradicional (con una distribución $t$ de Student simétrica).

\footnotetext{
${ }^{14}$ La excepción se refiere a la serie de rendimientos bursátiles de Perú. Las estimaciones de ambos modelos reportan valores similares para esta última serie.
} 
Intuitivamente, las volatilidades estimadas mediante el modelo TARCH propuesto probablemente reflejan los efectos de la crisis financiera global en los mercados latinoamericanos. Como es bien conocido, el periodo 2008-2009 se caracteriza por haber experimentado momentos de gran inestabilidad en los mercados internacionales. Particularmente, las altas volatilidades registradas podrían manifestar una eventual sincronización de los mercados de Argentina, Brasil, Chile, Colombia y México. En la práctica, esta situación podría haber tenido repercusiones para los inversionistas debido a que sus oportunidades para diversificar riesgos habrían sido relativamente escasas. 


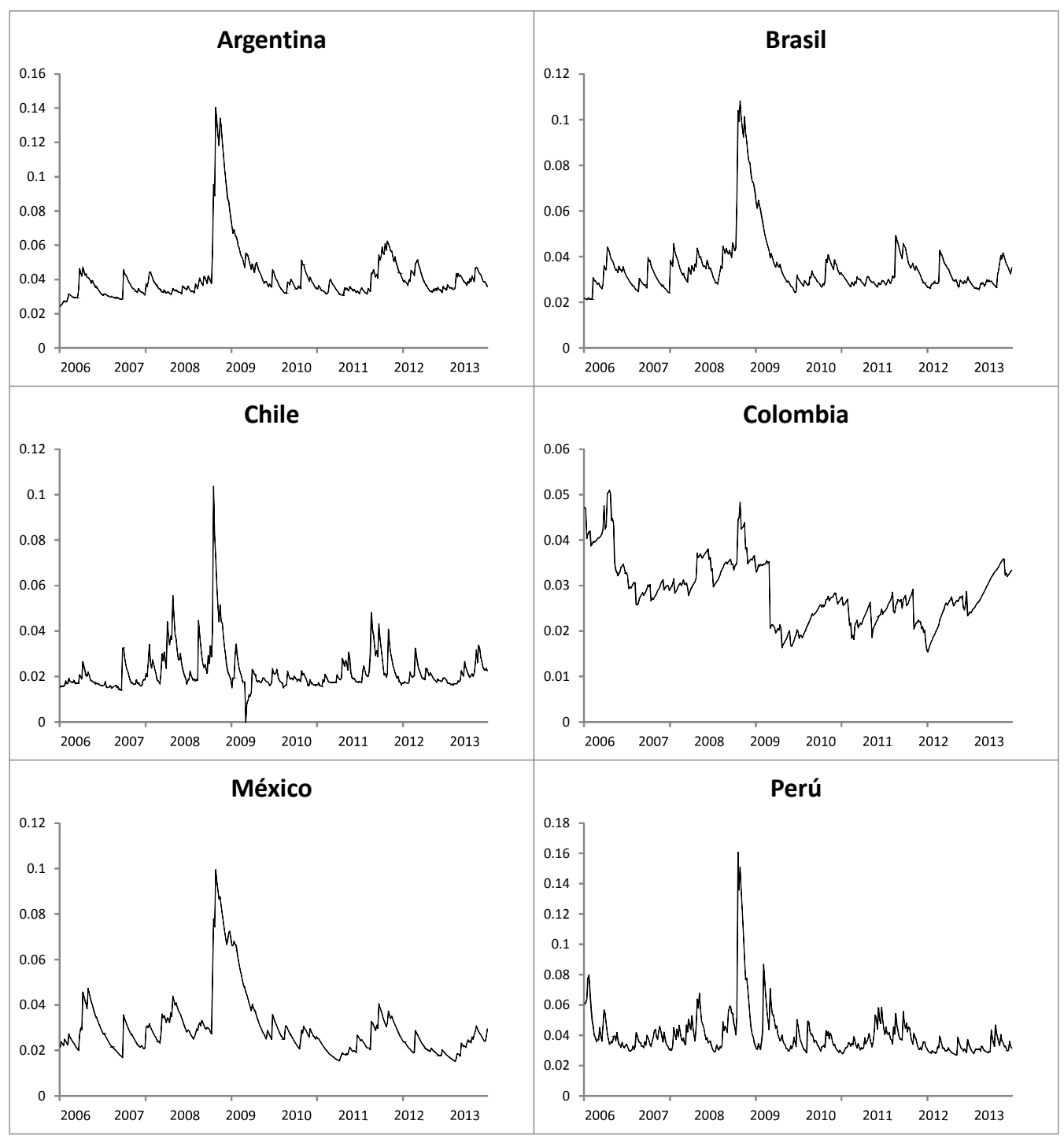

Figura 2. Gráficas de las volatilidades estimadas mediante el modelo TGARCH propuesto (con una distribución $t$ de Student asimétrica).

Finalmente, cabe enfatizar que el análisis sugiere que: 1) Cada una de las series de rendimientos bursátiles analizadas puede describirse mejor con el modelo TGARCH propuesto que con el modelo tradicional; 2) la hipótesis de racionalidad de Samuelson es consistente con la evidencia de los mercados de Argentina, Brasil, Chile, Colombia y México; 3) hay efectos apalancamiento en las series de rendimientos de dichos mercados; 
4) la hipótesis tradicional de racionalidad es consistente con la evidencia de Perú; y 5) las volatilidades estimadas mediante el modelo propuesto son mayores que las estimadas mediante el modelo TGARCH tradicional durante el periodo 2008-2009.

\section{Conclusiones y discusión}

En esta investigación se ha desarrollado un modelo $\mathrm{ARCH}$ de tipo TGARCH con una distribución t de Student asimétrica. El mismo se ha construido usando la metodología de Fernández y Steel (1998) y el modelo TGARCH tradicional desarrollado por Zakoian (1994). ${ }^{15}$ El modelo se caracteriza por tener efectos apalancamiento y perturbaciones que siguen una distribución de densidad asimétrica. El mismo se ha usado para modelar series bursátiles y para evaluar la validez de las hipótesis de racionalidad. Las series analizadas han sido las de rendimientos semanales de los índices de Argentina, Brasil, Chile, Colombia, México y Perú entre el 30 de diciembre de 2005 y el 6 de septiembre de 2013.

Los resultados empíricos del estudio sugieren que: 1) Cada una de las series de rendimientos bursátiles analizadas puede describirse adecuadamente con el modelo TGARCH propuesto; 2) la hipótesis de racionalidad de Samuelson es consistente con la evidencia de los mercados de Argentina, Brasil, Chile, Colombia y México; 3) hay efectos apalancamiento en las series de rendimientos de dichos mercados; 4) la hipótesis tradicional de racionalidad es consistente con la evidencia de Perú; y 5) las volatilidades estimadas mediante el modelo propuesto son mayores que las estimadas mediante el modelo TGARCH tradicional durante el periodo 2008-2009.

\footnotetext{
${ }^{15}$ El nombre TGARCH se deriva de las principales características del modelo. TGARCH es acrónimo de "Threshold Generalized Autoregressive Conditional Heteroscedasticity".
} 
Los resultados obtenidos tienen implicaciones empíricas y teóricas. Empíricamente, los resultados validan la conveniencia de usar modelos de la familia ARCH que asuman efectos apalancamiento y momentos de orden superior a la varianza para describir los rendimientos bursátiles. Por esa razón el modelo desarrollado pudiera ser útil para tomar decisiones de administración de riesgos, de inversión y de valuación de activos. Estos resultados también tienen implicaciones para la teoría de portafolios. Concretamente sugieren que los retornos bursátiles pueden no distribuirse normalmente y que las funciones de utilidad de los inversionistas pueden no ser cuadráticas.

Finalmente, no sobra enfatizar que el desarrollo de modelos de la familia ARCH con distribuciones de densidad asimétricas parece una línea de investigación muy promisoria. En este sentido, el desarrollo de versiones de los modelos EGARCH, PARCH y ACGARC con distribuciones asimétricas quizá debiera explorarse en estudios posteriores. ${ }^{16}$ Estos modelos son relevantes porque asumen efectos apalancamiento y porque generalizan a varios modelos usados comúnmente en la literatura. Muy probablemente, la investigación alrededor de estos modelos será de gran utilidad para entender la dinámica de las series económicas y financieras de las economías emergentes.

\footnotetext{
${ }^{16}$ Los nombres de los modelos de la familia ARCH se definen en términos de acrónimos que definen sus principales características. Así EGARCH es acrónimo de "Exponential Generalized Autoregressive Conditional Heteroscedasticity". PARCH es acrónimo de "Power Autoregressive Conditional Heteroscedasticity". ACGARCH es acrónimo de "Asymmetric Component Generalized Autoregressive Conditional Heteroscedasticity". Una descripción detallada de estos modelos puede hallarse en el trabajo de Bollerslev (2010).
} 


\section{REFERENCIAS}

Arditti, F.D. H. Levi, (1975), "Portfolio efficiency analysis in three moments: The multiperiod case", Journal of Finance, 30(3), 797-809

Black, F., (1976), "Studies on stock price volatility changes", Proceedings of the 1976 Meetings of the American Statistical Association, Business and Economics Statistics Section, 177-181

Bollerslev, T. (2010), “Glossary to ARCH (GARCH)”, en Bollerslev, T., Russell, J.R. y M.W. Watson, (eds.), Volatility and Time Series Econometrics: Essays in Honor of Robert Engle (Oxford University Press, Oxford), 137-163

Bonilla, C.A. y J.P. Sepulveda, (2011), "Stock returns in emerging markets and the use of GARCH models", Applied Economics Letters, 18(14), 1321-1325

Borch, K., (1969), “A note on uncertainty and indifference curves”, Review of Economic Studies, 36(105), 1-4

Canela, M.A. y E. Pedreira-Collazo, (2007), "Portfolio selection with skewness in emerging market industries", Emerging Markets Review, 8(3), 230-250

Chunhachinda, P., K. Dandapani, S. Hamid, y A. Prakash, (1997), "Portfolio selection and skewness: Evidence from international stock markets", Journal of Banking and Finance, 21(2), 143-167

Ding Z., C.W. Granger y R. F. Engle, (1993), “A long memory property of stock market returns and a new model”, Journal of Empirical Finance, 1(1), 83-106

Durán-Vázquez, R., A. Lorenzo-Valdés y A. Ruiz-Porras, (2013), “Un modelo GARCH con asimetría condicional autorregresiva para modelar series de tiempo: Una aplicación para los rendimientos del Índice de Precios y Cotizaciones de la BMV”, 
en Ortíz-Arango, F., López-Herrera, F. y F. Venegas Martínez, (coords.), Avances Recientes en Valuación de Activos y Administración de Riesgos, Vol. 4. (ESE-IPN, UNAM y Universidad Panamericana, México D.F.), 247-262

Elton, E.J., M.J. Gruber, S.J. Brown y W.N. Goetzmann, (2013), Modern Portfolio Theory and Investment Analysis, Novena edición, Hoboken, Estados Unidos, Wiley

Engle R.F., (2004), "Risk and volatility: Econometric models and financial practice", American Economic Review, 94(3), 405-420

Feldstein, M.S., (1969), "Mean-Variance analysis in the theory of liquidity preference and portfolio selection", Review of Economic Studies, 36(105), 5-12

Fernández, C. y M.F.J. Steel, (1998), “On Bayesian modeling of fat tails and skewness”, Journal of the American Statistical Association, 93(441), 359-371

Harvey, C. y A. Siddique, (1999), "Autoregressive conditional skewness", Journal of Financial and Quantitative Analysis, 34(4), 465-487

Harvey, C. y A. Siddique, (2000), "Conditional skewness in asset pricing tests", Journal of Finance, 55(3), 1263-1295

Jean, W.H., (1971), "The extension of portfolio analysis to three or more parameters", Journal of Financial and Quantitative Analysis, 6(1), 505-515

Johnson, C. y F. Soriano, (2003), "Volatilidad del mercado accionario y la Crisis Asiática: Evidencia internacional de asimetrías", El Trimestre Económico, 71(282), 355-388

Lambert, P. y S. Laurent (2001), "Modeling financial time series using GARCH-type models with a skewed student distribution for the innovations", Lovaina, Universite Catholique de Louvain-Institut de Statistique, Discussion Paper 0125

Lorenzo-Valdés, A. y A. Ruiz-Porras (2012), “Modelación de los rendimientos bursátiles mexicanos mediante los modelos TGARCH y EGARCH: Un estudio econométrico 
para 30 acciones y el Índice de Precios y Cotizaciones", en Coronado Ramírez S. y L. Gatica Arreola (coords.), Modelos no Lineales en Series Económicas y/o Financieras, (Universidad de Guadalajara, Guadalajara), 46-81

Markowitz, H. (1952), “Portfolio selection”, Journal of Finance, 7(1), 77-91

Mhiri, M. y JL. Prigent, (2010), "International portfolio optimization with higher moments", International Journal of Economics and Finance, 2(5), 157-169

Nelson, D.B. (1991), "Conditional heteroskedasticity in asset returns: A new approach", Econometrica, 59(2), 347-370

Prakash, A.J., CH. Chang y T.E. Pactwa, (2003), "Selecting a portfolio with skewness: Recent evidence from US, European and Latin American equity markets”, Journal of Banking and Finance, 27(7), 1375-1390

Samuelson, P.A., (1970), "The fundamental approximation theory of portfolio analysis in terms of means, variances and higher moments", Review of Economic Studies, $37(4), 537-542$

Sharpe, W.F., (1964), "Capital asset prices: A theory of market equilibrium under conditions of risk", Journal of Finance, 19(3), 425-442

Tobin, J., (1958), "Liquidity preference as behavior towards risk", Review of Economic Studies, 25(2), 65-86

Tsiang, S.C., (1974), "The rationale of the mean-standard deviation analysis, skewness preference and the demand for money", American Economic Review, 62(3), 354371

Xiong, J.X. y T.M. Idzorek, (2011), “The impact of skewness and fat tails on the asset allocation decision”, Financial Analyst Journal, 67(2), 23-35 
Zakoian, JM., (1994), “Threshold heteroskedastic models”, Journal of Economic Dynamics and Control, 18(5), 931-955 